\title{
Tanshinone IIA inhibits the dihydrotestosterone-induced secretion of lipids and activation of sterol regulatory element binding protein-1 in HaCaT cells
}

\author{
DONG-YAN SONG ${ }^{1,2}$, QIU-HONG HUANG ${ }^{1}$, BING-RONG ZHOU ${ }^{1}$, YANG XU ${ }^{1}$, \\ ZHI-QIANG YIN ${ }^{1}$, FELICIA PERMATASARI ${ }^{1}$ and DAN LUO ${ }^{1}$ \\ ${ }^{1}$ Department of Dermatology, The First Affiliated Hospital of Nanjing Medical University, Nanjing 210029; \\ ${ }^{2}$ Department of Dermatology, The Affiliated Jiangyin Hospital of Southeast \\ University Medical College, Jiangyin 214400, P.R. China
}

Received March 12, 2012; Accepted May 10, 2012

DOI: $10.3892 / \mathrm{etm} .2012 .586$

\begin{abstract}
To study the effects and mechanisms of Tanshinone IIA (Tan IIA) on the dihydrotestosterone (DHT)-induced expression of sterol regulatory element binding protein-1 (SREBP-1), the synthesis and secretion of lipids in $\mathrm{HaCaT}$ cells were examined. HaCaT cells were treated with DHT and Tan IIA at different concentrations. Real-time PCR was used to detect the expression of SREBP-1c, fatty acid synthase (FAS), acyl-CoA synthetase (ACS), stearoylCoA desaturase (SCD) and HMG-CoA reductase (HMGCR) mRNA in HaCaT cells. Western blotting was used to analyze the protein expression of SREBP-1 and phosphorylation of Akt. Flow cytometry and Nile red staining were used to detect the synthesis and secretion of lipids in HaCaT cells. We observed that Tan IIA inhibited the DHT-induced expression of SREBP-1 and p-AKT in HaCaT cells, which produced an effect similar to that of LY294002. Tan IIA significantly inhibited the transcription of lipid synthesis-related genes and decreased lipid secretion in $\mathrm{HaCaT}$ cells. In conclusion, Tan IIA downregulates the expression of lipid synthesis-related genes and decreases lipid secretion in HaCaT cells, which is correlated with the inhibitory effect on the DHT-induced mRNA and protein expression of SREBP-1 in HaCaT cells.
\end{abstract}

\section{Introduction}

The excessive secretion of sebum on skin is a significant factor in various skin diseases (including acne and seborrheic dermatitis) $(1,2)$. Medication remains the main method of treatment

Correspondence to: Professor Dan Luo, Department of Dermatology, The First Affiliated Hospital of Nanjing Medical University, Guangzhau Road 300, Jiangsu, Nanjing 210029, P.R. China E-mail: daniluo2005@yahoo.com.cn

Key words: Tanshinone IIA, dihydrotestosterone, HaCaT cells, sterol regulatory element binding protein-1 for diseases in which excessive sebum is secreted. Cis-retinoic acid has been shown to inhibit the proliferation of sebaceous gland cells and reduce lipid secretion (3-6), and has been suggested to be the most effective anti-acne drug currently in use. However, studies have shown that cis-retinoic danshen/ tanshin acid may induce several side effects, including cerebral damage $(7,8)$, abnormal function of the skin barrier (9), intestinal mucosa damage (10), teratogenicity (11) and psychological problems (12), therefore there are limitations to its clinical use. Thus, identifying more effective and safer drugs to treat sebum secretion is clearly of practical significance.

Tanshinone is the main substance among the fat-soluble extracts obtained from the Chinese herbal medicine danshen/ tanshin, which comes from a member of the Labiatae family commonly used for the treatment of cardio-cerebrovascular diseases. There have been numerous Chinese clinical studies concerning its use in the treatment of acne, seborrheic dermatitis and diseases of excessive sebum secretion (13-15). To date, more than ten monomers have been identified, among which Tanshinone IIA (Tan IIA) has been the most extensively studied. A wide range of pharmacological uses have been reported for Tan IIA, for example, neuroprotective effects (16), prevention of cardiac hypertrophy (17), antitumor (18), anti-atherosclerosis (19) and anti-inflammatory (20) effects and protection of vascular endothelial cells (21). In addition, a number of Chinese studies have reported that Tan IIA is effective against the secretion of sebum, either by inhibiting the proliferation of sebaceous glands and the synthesis of lipids, or by indirectly downregulating the expression of mRNA of androgen receptors in sebaceous gland cells (22). Our previous studies have demonstrated that the topical application of Tan IIA sodium sulfonate is able to significantly inhibit the proliferation of Syrian hamster sebaceous glands (23). However, further study is required to identify the underlying mechanisms.

In recent years, the sterol regulatory element binding protein (SREBP) pathway has been a main focus in studies of the mechanism of lipid secretion. SREBPs are one of the most significant regulatory factors for lipid synthesis in animals and regulate the synthesis of cholesterol, fatty acids and triglycerides through regulating the gene transcription of lipid 
Table I. Primers and amplified products.

Genes

Primers

Products (bp)

SREBP-1c

FAS

ACS

SCD

HMGCR

Actin
Forward: 5' GGAGCCATGGATTGCACTTT 3'

Reverse: 5' TCAAATAGGCCAGGGAAGTCA 3'

Forward: 5' CAGGCACACACGATGGAC 3'

Reverse: 5' CGGAGTGAATCTGGGTTGAT 3'

Forward: 5' CCCAGTTTATCCCAATGCTG 3'

Reverse: 5' GGGCGCCATAGAACTGATT 3'

Forward: 5' CCGGGAGAATATCCTGGTTT 3'

Reverse: 5' GCGGTACTCACTGGCAGAGT 3'

Forward: 5' TGGCTCTTTCAGAGAGGTCTCA 3'

Reverse: 5' TGCCTTCAGAGGTGAGCTGTA 3'

Forward: 5' GCAGAAGGAGATCACAGCCCT 3'

Reverse: 5' GCTGATCCACATCTGCTGGAA 3'
77

92

74

97

158

136

FAS, fatty acid synthase; ACS, acyl-CoA synthetase; SCD, stearoyl-CoA desaturase; HMGCR, HMG-CoA reductase; SREBP-1, sterol regulatory element binding protein-1.

synthesis-related enzymes (24-26). Rosignoli et al (27) found that androgenic hormones regulate the gene transcription of lipid synthesis-related enzymes through activating the SREBP pathway in sebaceous gland tissues, as a result of increased synthesis and secretion of lipid. Apart from sebaceous glands, keratinocytes (KCs) are another significant source of lipids on the skin surface. Harrison et al (28) found that SREBP-1c was expressed in KCs and played a role in lipid synthesis. In this study, we observed that Tan IIA inhibits dihydrotestosterone (DHT)-induced lipid synthesis and secretion in HaCaT cells, which may occur via blocking of the activity of the PI3K/Akt pathway. This is the first study on the mechanism of the inhibitory effect of Tan IIA on lipid secretion in HaCaT cells.

\section{Materials and methods}

Drugs. DHT powder was purchased from Sigma Co. Ltd. (St. Louis, MO, USA) and Tan IIA powder was purchased from Nanjing Zelang Medical Technological Co. Ltd. (Jiangsu, China). These were diluted with DMSO prior to use, prepared in serum-free medium at the required concentrations and subpackaged by filtration sterilization. Anti-SREBP-1 (polyclonal) was purchased from Beijing Boao Bio-tech Co. Ltd., (Beijing, China); rabbit anti-phospho-Akt (Ser473; monoclonal) was purchased from Cell Signaling Technology, Inc. (Danvers, MA, USA); mouse/rabbit anti-actin, PI3K inhibitor LY294002 and BCA Protein Assay kit were purchased from Beyotime Institute of Biotechnology (Haimen, Jiangsu, China); TRIzol was purchased from Invitrogen (Carlsbad, CA, USA); real-time PCR Assay kits were purchased from Nanjing KGI Bioteknologi Development Co, Ltd. (Nanjing, China); Nile red powder was purchased from Shanghai XinRan Bio-tech Co. Ltd. (Shanghai, China), diluted with methanol and stored at a concentration of $10 \mu \mathrm{g} / \mathrm{ml}$ at $4^{\circ} \mathrm{C}$, away from light.

Cell culture. Cells of the HaCaT KC cell line were cultured in a cell incubator at $37^{\circ} \mathrm{C}$ in $5 \% \mathrm{CO}_{2}$, in DMEM medium containing $10 \%$ fetal bovine serum, $1 \%$ penicillin and streptomycin. After the cells became polygonal, arranged as a single layer, they were vaccinated at a density of $1 \times 10^{9}$ cells/1 with $0.25 \%$ trypsin solution. The cultured cells were used for experimentation when they adhered to the culture plate and the confluence reached $70-80 \%$.

Experimental grouping and treatment of cells. The cells were cultured in serum-free medium and, after growing synchronously for $24 \mathrm{~h}$, they were divided randomly into five groups: i) control; ii) DHT, administered DHT at a concentration of $100 \mathrm{nmol} / \mathrm{l}$; iii) Tan IIA $(1.25 \mu \mathrm{mol} / \mathrm{l})+$ DHT; iv) Tan IIA $(2.5 \mu \mathrm{mol} / \mathrm{l})+$ DHT; v) Tan IIA $(5 \mu \mathrm{mol} / \mathrm{l})+$ DHT. The cells were treated with Tan IIA for $24 \mathrm{~h}$ before adding $100 \mathrm{nmol} / \mathrm{l}$ DHT and were then cultured for a further $24 \mathrm{~h}$ prior to use.

To observe the effect of Tan IIA and LY294002 on DHT-induced lipid synthesis and lipid synthetase-related genes in $\mathrm{HaCaT}$ cells, the cells were cultured in serum-free medium, and after growing synchronously for $24 \mathrm{~h}$ they were divided randomly into four groups: i) control; ii) DHT, administered DHT at a concentration of $100 \mathrm{nmol} / \mathrm{l}$; iii) an LY294002 + DHT group, pre-treated with LY294002 at $50 \mu \mathrm{M}$ for $40 \mathrm{~min}$, then with DHT added at concentration of $100 \mathrm{nmol} / \mathrm{L}$, and then cultured for a further $24 \mathrm{~h}$ prior to use; iv) a Tan IIA $2.5 \mu \mathrm{mol} / 1+$ DHT group in which the Tan IIA was pre-treated for $24 \mathrm{~h}$ prior to being added to $100 \mathrm{nmol} / \mathrm{l} \mathrm{DHT}$, and then cultured for a further $24 \mathrm{~h}$ prior to use.

Real-time PCR detection of the expression of SREBP-1c mRNA and the expression of lipogenic enzyme [fatty acid synthase (FAS), acyl-CoA synthetase (ACS), stearoyl-CoA synthetase (SCD) and HMG-CoA reductase (HMGCR)] mRNA in HaCaT cells. The cells were grouped and TRIzol was added to break down the cells, followed by extraction of total RNA, measurement of concentration and then measurement of purity. After ensuring that the quality met the requirements of the experiment, cDNA was obtained by reverse transcription. cDNA 


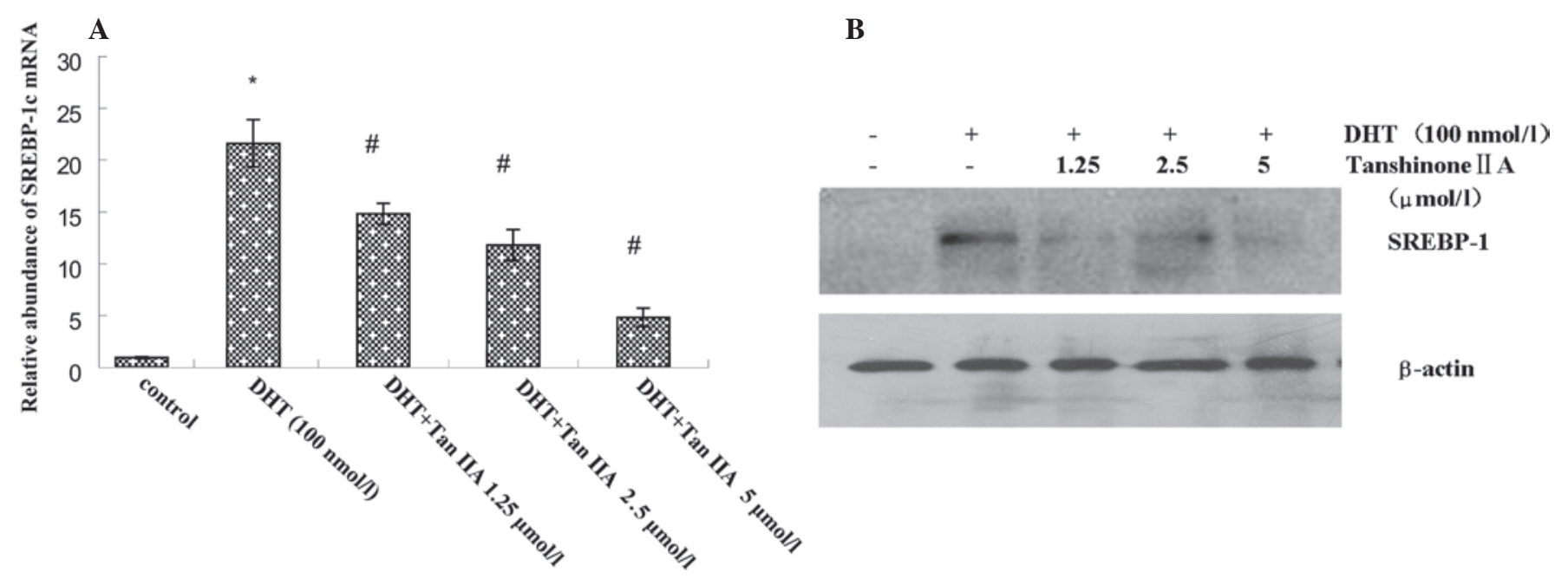

Figure 1. The effect of Tan IIA on the DHT-activated SREBP-1 pathway in HaCaT cells. (A) Tan IIA inhibits the DHT-induced expression of SREBP-1c mRNA in HaCaT cells. (B) Tan IIA inhibits the DHT-induced protein expression of SREBP-1 in HaCaT cells. "P<0.01 vs. blank control group; "P<0.05 vs. DHT group. SREBP-1, sterol regulatory element binding protein-1; DHT, dihydrotestosterone; Tan IIA, Tanshinone IIA.

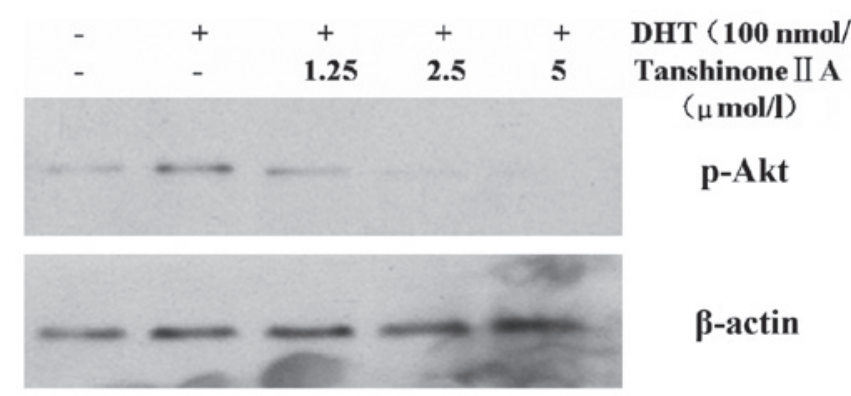

Figure 2. Effect of Tan IIA on the DHT-activated PI3K/Akt pathway in $\mathrm{HaCaT}$ cells. Tan IIA inhibits the DHT-induced protein expression of p-Akt in HaCaT cells. Tan IIA, Tanshinone IIA; DHT, dihydrotestosterone.

was diluted 10 times and amplified in a $20-\mu 1$ reaction system. Primers were synthesized by Nanjing Kaiji Bio-tech Co, Ltd. (Table I). The amplification conditions were as follows: pre-denaturation at $95^{\circ} \mathrm{C}$ for $5 \mathrm{~min}$, entering reaction cycles, denaturation at $95^{\circ} \mathrm{C}$ for $15 \mathrm{~min}$, annealing for $30 \mathrm{sec}$ at $60^{\circ} \mathrm{C}$, extending for $30 \mathrm{sec}$ at $72^{\circ} \mathrm{C}$ and maintained at $72^{\circ} \mathrm{C}$ for $10 \mathrm{~min}$ following 40 cycles.

Western blot tests for the protein expression of SREBP-1 and $p$-Akt. The cells were treated according to group. The culture solution was discarded, the cells were washed three times with PBS solution pre-cooled to $4^{\circ} \mathrm{C}, 300 \mu \mathrm{l}$ cell degradation solution containing protease inhibitor was added, then the mixture was placed on ice for $15 \mathrm{~min}$ and, following cell detachment, centrifugal separation was conducted at $4^{\circ} \mathrm{C}$ at $12,000 \mathrm{rpm}$. The upper layer of the solution was tested for protein using the BCA method. A 30- $\mu \mathrm{g}$ sample of protein was taken from each group for SDS-PAGE, protein was transferred onto PVDF films and covered with a 5\% BSA blocking buffer at $37^{\circ} \mathrm{C}$ for $1 \mathrm{~h}$. The primary antibody was added according to the cell group (rabbit antibody SREBP-1, diluted at 1:500; rabbit antibody phospho-Akt, diluted at 1:1,000; actin antibody diluted at $1: 1,000)$ and the cells were incubated at $4^{\circ} \mathrm{C}$ throughout the night, washed prior to incubation with the secondary antibody, which was diluted at 1:1,000, and marked by horseradish peroxidase at $37^{\circ} \mathrm{C}$ for $40 \mathrm{~min}$. ECL detection reagent was added for $5 \mathrm{~min}$. Finally, a fixation procedure was conducted.

Detecting the effects of Tan IIA on the DHT-induced synthesis of lipids in $\mathrm{HaCaT}$ cells using flow cytometry. $\mathrm{HaCaT}$ cells at the exponential phase of growth were inoculated in 6-well plates, $3 \times 10^{4}$ cells/well and cultured for $24 \mathrm{~h}$. The HaCaT cells were treated according to group and $0.25 \%$ trypsin solution containing $0.02 \%$ EDTA was added. The degradation process was terminated with $10 \%$ fetal bovine serum medium. The cells were washed twice with PBS, a single-cell suspension was prepared (in PBS), $100 \mathrm{ng} / \mathrm{ml}$ Nile red fluorescent dye was added, samples were incubated at room temperature for 15 min, filtered with 300 mesh nylon membrane and flow cytometry was then used to test 10,000 cells from each sample. The average fluorescence intensity of every cell was determined, with an excitation wavelength at $485 \mathrm{~nm}$ and emission wavelength at $565 \mathrm{~nm}$.

Statistical analysis. SPSS 13.0 software (SPSS, Inc., Chicago, IL, USA) was used for data analysis, and the form of mean \pm standard deviation was used to indicate measurement data. A t-test was used for inter-group comparison, and $\mathrm{P}<0.05$ was considered to indicate a statistically significant result.

\section{Results}

Effect of Tan IIA on the DHT-activated SREBP-1 pathway in HaCaT cells. Compared with the low expression of SREBP-1c mRNA and SREBP-1 protein in the untreated control group, significantly higher levels of expression of SREBP-1c mRNA and SREBP-1 protein were observed in DHT-treated HaCaT cells. Different concentrations of Tan IIA were added prior to DHT treatment, resulting in a decrease in SREBP-1c mRNA and SREBP-1 protein expression in a concentrationdependent manner (Fig. 1). Overall, in quantitative analysis, compared with the unexposed control epidermis, after $24 \mathrm{~h}$ 


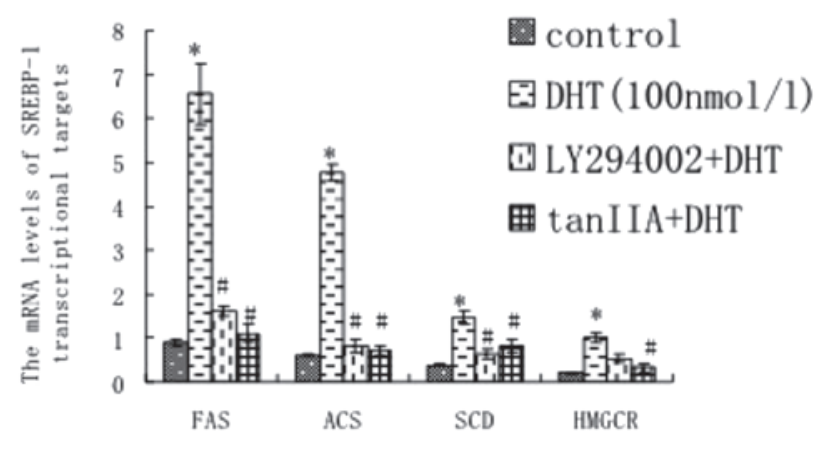

Figure 3. Effect of Tan IIA and LY294002 on DHT-induced expression of lipid synthetase-related genes, as tested by real-time PCR, in HaCaT cells. ${ }^{*} \mathrm{P}<0.01$ vs. blank control group; ${ }^{\#} \mathrm{P}<0.05$ vs. DHT group. Tan IIA, Tanshinone IIA; FAS, fatty acid synthase; ACS, acyl-CoA synthetase; SCD, stearoyl-CoA desaturase; HMGCR, HMG-CoA reductase; SREBP-1, sterol regulatory element binding protein-1; DHT, dihydrotestosterone.

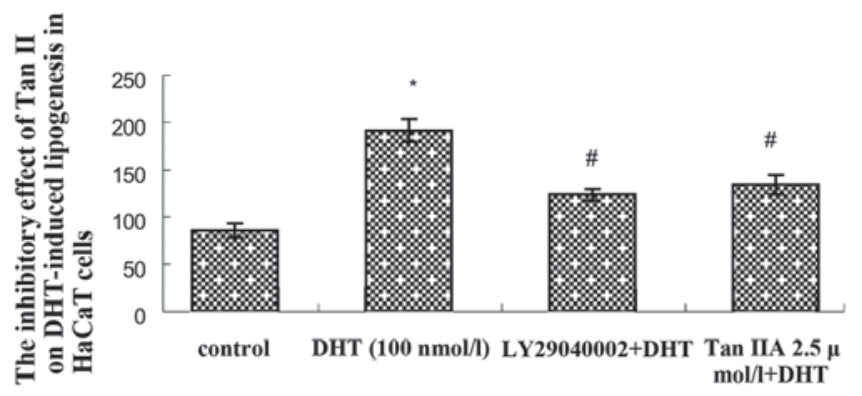

Figure 4. Effect of Tan IIA and LY294002 on DHT-induced lipid synthesis in $\mathrm{HaCaT}$ cells. ${ }^{*} \mathrm{P}<0.01$ vs. blank control group; ${ }^{*} \mathrm{P}<0.05$ vs. DHT group. Tan IIA, Tanshinone IIA; DHT, dihydrotestosterone.

exposure to DHT, the SREBP-1 protein accumulation was $21.71 \%$. This was decreased by pre-application of different concentrations of Tan IIA $(1.25,2.5$ and $5 \mu \mathrm{mol} / 1)$ to 6.34 , $16.82(\mathrm{P}<0.05)$ and $10.17 \%$ SREBP-1 protein expression, respectively.

Effect of Tan IIA on the DHT-activated p-Akt in HaCaT cells. Compared with a low protein expression for p-Akt in the untreated control group, a significantly higher expression of p-Akt protein was observed in DHT-treated HaCaT cells. A different concentration of Tan IIA was added prior to DHT treatment, however, this resulted in a decrease in p-Akt protein expression in a concentration-dependent manner (Fig. 2). Overall, in quantitative analysis, compared with the unexposed control epidermis, after $24 \mathrm{~h}$ exposure to DHT the p-Akt protein accumulation was $18.32 \%$. This was decreased by pre-application of different concentrations of Tan IIA (1.25, 2.5 and $5 \mu \mathrm{mol} / \mathrm{l})$ to $11.22,6.18(\mathrm{P}<0.05)$ and $2.43 \% \mathrm{p}$-Akt protein expression, respectively.

Effect of Tan IIA and LY294002 on the expression of lipid synthetase-related genes in $\mathrm{HaCaT}$ cells. According to the results of real-time PCR tests (Fig. 3), DHT $(100 \mathrm{nmol} / \mathrm{l})$ increased the transcription of FAS, ACS, SCD and HMGCR $(\mathrm{P}<0.01)$ when compared with the untreated control group. Following pre-treatment with LY294002 for $40 \mathrm{~min}$, the induction effect of DHT on the expression of FAS, ACS, SCD and HMGCR was significantly inhibited $(\mathrm{P}<0.01)$. Tan IIA played a role similar to LY294002 and significantly inhibited the SREBP-1-regulated gene transcription of lipid synthetase $(\mathrm{P}<0.01)$, which was consistent with the inhibitory effects of Tanshinone on SREBP-1c.

Effect of Tan IIA on DHT-induced lipid synthesis in HaCaT cells. The results of flow cytometry showed that the average fluorescence intensity of the DHT group was significantly increased $(\mathrm{P}<0.05)$ compared with the untreated control group. The average fluorescence intensity of the LY294002+DHT group and the Tan IIA+DHT group were decreased compared with the blank control group $(\mathrm{P}<0.05$; Fig. 4). The results above indicate that Tan IIA has an inhibitory effect on the lipid synthesis of HaCaT cells induced by DHT, an effect similar to that of LY294002.

\section{Discussion}

In recent years, much attention has been focused on intracellular signal transduction mediating the activation of the SREBP pathway, which plays a role in lipogenesis. The PI3K/ Akt signaling and mitogen-activated protein kinase (MAPK) pathways play critical roles in mediating the activation of the SREBP pathway in different cell types (29-32). Previous studies have indicated that the activation of Akt is involved in the transport of the SREBP cleavage-activating protein (SCAP)/SREBP complex from the endoplasmic reticulum to the Golgi (33). This is a major regulatory step in SREBP activity. In the present study, we demonstrated for the first time that DHT-induced SREBP-1 expression is regulated by the PI3K/Akt pathway. Our data indicate that DHT increases the amount of cleaved (mature) SREBP protein and that this increase is inhibited in the presence of LY294002. This suggests that, in addition to possible transcriptional and translational control, Akt activation may also affect SREBP processing in $\mathrm{HaCaT}$ cells. Akt activation has also been shown to increase the expression of lipogenic genes (34). The conclusion is further supported by our observation that the inhibition of Akt activation by LY294002 blocks the increase in mRNA expression of lipogenic genes and lipogenesis induced by SREBP-1.

Chinese clinical studies have shown that Tanshinone has significant effects on reducing sebum secretion and is used to treat acne and seborrheic dermatitis (13-15). Tan IIA is the most effective pharmacological ingredient of Tanshinone and has become a popular topic of research, with extensive studies in numerous fields of pharmacological effects, including anti-oxidant, anti-inflammatory and antibiotic properties, liver-protection and the ability to prevent tumors and improve circulation. However, no studies concerning the specific pathways and mechanisms of the anti-lipid effects of Tan IIA have been performed. In the present study, we found that Tan IIA significantly inhibits the expression of SREBP-1 induced by DHT, downregulates the transcription of enzyme genes associated with lipid synthesis, including FAS, ACS, SCD and HMGCR, and significantly reduces lipid production and secretion in $\mathrm{HaCaT}$ cells. The results of this study demonstrate that Tan IIA is able to counter lipid secretion in KCs, a property associated with its regulation of the expression of SREBP-1. In 
addition, we found that Tan IIA is able to produce an inhibitory effect on the SREBP-1 pathway through the PI3K/Akt signaling pathway; its inhibitory effect on the DHT-induced expression of SREBP-1 and lipid synthesis in HaCaT cells is identical to the effect of the PI3K inhibitor LY294002. Therefore, we consider that in the suppression of skin lipid secretion, Tan IIA may produce effects similar to a PI3K inhibitor.

In conclusion, this study indicates that Tan IIA is capable of inhibiting lipid secretion caused by an excess of androgenic hormones and that it may be used for the treatment of skin diseases including acne and seborrheic dermatitis in which an excessive amount of lipid is secreted.

\section{Acknowledgements}

This study was supported by grants from the China National Natural Science Foundation (81000700, 81171518). We appreciate the assistance of NT Pharma (China) Investment Co., Ltd. during the editing process.

\section{References}

1. Zouboulis CC: Acne and sebaceous gland function. Clin Dermatol 22: 360-366, 2004

2. Plewig G: How acne vulgaris develops. Hautarzt 61: 99-100, 102-104, 106 (In German).

3. McDonald SK, Goh MS and Chong AH: Successful treatment of cyclosporine-induced sebaceous hyperplasia with oral isotretinoin in two renal transplant recipients. Australas J Dermatol 52: 227-230, 2011.

4. Orfanos CE and Zouboulis CC: Oral retinoids in the treatment of seborrhoea and acne. Dermatology 196: 140-147, 1998.

5. Doran TI and Shapiro SS: Retinoid effects on sebocyte proliferation. Methods Enzymol 190: 334-338, 1990.

6. De Marchi MA, Maranhão RC, Brandizzi LI and Souza DR Effects of isotretinoin on the metabolism of triglyceride-rich lipoproteins and on the lipid profile in patients with acne. Arch Dermatol Res 297: 403-408, 2006

7. Wong A, Williams M and Gibb W: Isotretinoin-induced encephalopathy. J Dermatolog Treat 21: 361-362, 2010.

8. Yaman M, Albayram S, Altintas A, et al: A cerebellar demyelinating lesion following treatment of acne with isotretinoin. Clin Exp Dermatol 33: 118-121, 2008.

9. Tinoco MP, Tamler C, Maciel G, et al: Pyoderma gangrenosum following isotretinoin therapy for acne nodulocystic. Int J Dermatol 47: 953-956, 2008.

10. Papageorgiou NP, Altman A and Shoenfeld Y: Inflammatory bowel disease: adverse effect of isotretinoin. Isr Med Assoc J 11: 505-506, 2009.

11. Malvasi A, Tinelli A, Buia A and De Luca GF: Possible long-term teratogenic effect of isotretinoin in pregnancy. Eur Rev Med Pharmacol Sci 13: 393-396, 2009.

12. Azoulay L, Blais L, Koren G, et al: Isotretinoin and the risk of depression in patients with acne vulgaris: a case-crossover study. J Clin Psychiatry 69: 526-532, 2008.

13. Gao YG, Wang LZ and Tang JX: Sex hormone-like activity of Tanshinone (author's transl). Zhongguo Yi Xue Ke Xue Yuan Xue Bao 2: 189-191, 1980 (In Chinese).

14. Wang DB and Liu AS: Tanshinone in the treatment of acne (a primary report of 20 cases) (author's transl). Zhongguo Yi Xue Ke Xue Yuan Xue Bao 2: 187-188, 1980 (In Chinese).

15. Wang DB: Tanshinone in the treatment of acne. J Tradit Chin Med 3: 227-228, 1983.
16. Hei M, Luo Y, Zhang X and Liu F: Tanshinone IIa alleviates the biochemical changes associated with hypoxic ischemic brain damage in a rat model. Phytother Res 25: 1865-1869, 2011.

17. Tan X, Li J, Wang X, et al: Tanshinone IIA protects against cardiac hypertrophy via inhibiting calcineurin/NFATc3 pathway. Int J Biol Sci 7: 383-389, 2011.

18. Cheng CY and Su CC: Tanshinone IIA may inhibit the growth of small cell lung cancer H146 cells by up-regulating the Bax/ $\mathrm{Bcl}-2$ ratio and decreasing mitochondrial membrane potential. Mol Med Report 3: 645-650, 2010.

19. Bian Z, Li LM, Tang R, et al: Identification of mouse liver mitochondria-associated miRNAs and their potential biological functions. Cell Res 20: 1076-1078, 2010.

20. Dong X, Dong J, Zhang R, et al: Anti-inflammatory effects of tanshinone IIA on radiation-induced microglia BV-2 cells inflammatory response. Cancer Biother Radiopharm 24: 681-687, 2009.

21. Lin R, Wang WR, Liu JT, et al: Protective effect of tanshinone IIA on human umbilical vein endothelial cell injured by hydrogen peroxide and its mechanism. J Ethnopharmacol 108: 217-222, 2006.

22. Qiang J, Xingping Y, Jihai S, et al: Effects of Cryptotanshinone and Tanshinone A on proliferation, lipid synthesis and expression of androgen receptor mRNA in human sebocytes in vitro. Chin J Dermatol 38: 98-101, 2005 (In Chinese).

23. Huang Q, Zhou B, Guo X, et al: Sulfotanshinone sodium suppresses sebaceous hyperplasia in Syrian hamsters. Chin J Dermatol 44: 643-645, 2011 (In Chinese).

24. Horton JD and Shimomura I: Sterol regulatory element-binding proteins: activators of cholesterol and fatty acid biosynthesis. Curr Opin Lipidol 10: 143-150, 1999.

25. Shimano H: Sterol regulatory element-binding proteins (SREBPs): transcriptional regulators of lipid synthetic genes. Prog Lipid Res 40: 439-452, 2001.

26. Eberlé D, Hegarty B, Bossard P, et al: SREBP transcription factors: master regulators of lipid homeostasis. Biochimie 86: 839-848, 2004

27. Rosignoli C, Nicolas JC, Jomard A and Michel S: Involvement of the SREBP pathway in the mode of action of androgens in sebaceous glands in vivo. Exp Dermatol 12: 480-489, 2003.

28. Harrison WJ, Bull JJ, Seltmann H, et al: Expression of lipogenic factors galectin-12, resistin, SREBP-1, and SCD in human sebaceous glands and cultured sebocytes. J Invest Dermatol 127: 1309-1317, 2007.

29. Smith TM, Gilliland K, Clawson GA and Thiboutot D: IGF-1 induces SREBP-1 expression and lipogenesis in SEB-1 sebocytes via activation of the phosphoinositide 3-kinase/Akt pathway. J Invest Dermatol 128: 1286-1293, 2008.

30. Chang Y, Wang J, Lu X, et al: KGF induces lipogenic genes through a PI3K and JNK/SREBP-1 pathway in H292 cells. J Lipid Res 46: 2624-2635, 2005.

31. Hao J, Liu S, Zhao S, et al: PI3K/Akt pathway mediates high glucose-induced lipogenesis and extracellular matrix accumulation in HKC cells through regulation of SREBP-1 and TGF- $\beta 1$. Histochem Cell Biol 135: 173-181, 2011.

32. Yang YA, Han WF, Morin PJ, et al: Activation of fatty acid synthesis during neoplastic transformation: role of mitogenactivated protein kinase and phosphatidylinositol 3-kinase. Exp Cell Res 279: 80-90, 2002.

33. Du X, Kristiana I, Wong $\mathrm{J}$ and Brown AJ: Involvement of Akt in ER-to-Golgi transport of SCAP/SREBP: a link between a key cell proliferative pathway and membrane synthesis. Mol Biol Cell 17: 2735-2745, 2006.

34. Porstmann T, Griffiths B, Chung YL, et al: PKB/Akt induces transcription of enzymes involved in cholesterol and fatty acid biosynthesis via activation of SREBP. Oncogene 24: 6465-6481, 2005. 